\section{Pupillary response and galvanic skin response during an imagery task'}

FRANK D. COLMAN and ALLAN PAIVIO, The University of Western Ontario, London, Ont., Canada

Pupillary responses (PR) and galvanic skin responses (GSR) were continuously monitored while Ss attempted to generate images suggested by both concrete and abstract words. Ss were required to press a key when an image occurred or at the end of the "image" period if they were unable to generate an image. The results showed a significant difference between concrete and abstract nouns with regard to pupil size, time to maximum pupil size, time to maximum GSR, and key-press latency. These results suggest that the pupillary response, especially its latency, may be a more sensitive peripheral response than GSR during cognitive tasks.

The purpose of this study was to compare pupillary dilation and GSR during an imagery task in order to determine the relative sensitivity of each measure to the subtle differences in difficulty of such a cognitive task.

Recent evidence has suggested that pupillary dilation is related to the difficulty of cognitive tasks. Hess \& Polt (1964) recorded changes in pupil size during the solution of simple multiplication problems. They found that the amount of dilation increased with the difficulty of the problem, the pupil reaching a maximum diameter just before an answer was given. Paivio \& Simpson (1966) found that significantly greater dilation occurred to abstract than to concrete nouns during an imagery task in which Ss were asked to form images or mental pictures to the stimulus words. This experiment provided physiological evidence consistent with behavioral latency data
(Paivio, 1966; Yuille \& Paivio, 1967) which indicated that imaging to abstract words was more difficult than imaging to concrete words. Kahneman \& Beatty (1966) have also shown that greater pupillary dilation is associated with more difficult cognitive tasks (i.e., transformation as opposed to simple repetition).

Another physiological index often investigated during cognitive tasks is the galvanic skin response (GSR). Investigations using the GSR as an indicator of task difficulty, however, have produced conflicting results. Although Davis (1934) reported "no discernable decrease in the resistance change accompanying mental work when the performance is repeated," there was an increase when the Ss were asked on the final day to try to surpass previous accomplishments. This result suggests that the GSR is perhaps reflecting motivational or attentional factors. GSR responsivity has been found to correlate positively with a number of tasks, including retention and recall (Silverman, Cohen, \& Schmavonian, 1959). However, Berry (1962a) reported that only moderate levels of skin conductance in the first minute of learning and recall periods were related to better recall. Berry (1962b) concluded that changes in arithmetic task (addition) difficulty had no significant effect on skin conductance levels, which contrasts with Kahneman \& Beatty (1966) results with pupillary dilation.

\section{APPARATUS AND MATERIALS}

The apparatus used to record pupillary dilation was similar to that described by Hess (1965). It consists of a box-like compartment with a screen at one end and a face-support surrounding an opening at the other end for the $S$ to view the screen. A $16 \mathrm{H}$ Bolex movie camera, mounted on the

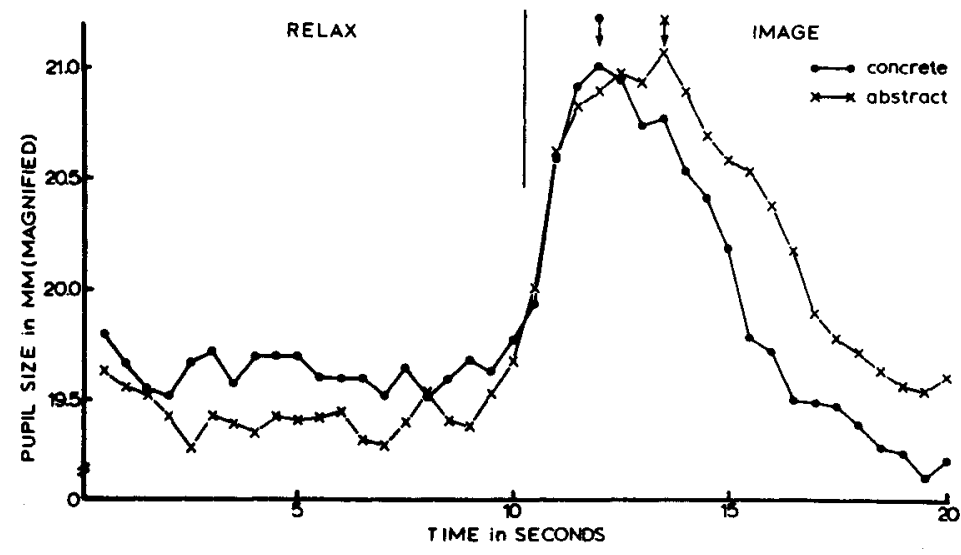

right-hand side of the box, protruded through an opening into the box and photographed the S's eye as reflected from a mirror inside the box. The camera was driven at a filming speed of two frames per second by a $120-$ rpm motor. A $25-W$ red bulb in a reflector inside the box, approximately 8 in. from the S's eye, provided the illumination and served as an infrared source for the Kodak high-speed infrared film that was used. A telegraph key was placed by the right of the pupillary apparatus near the S's right hand.

The GSR was obtained from two Grass E5G cup-type electrodes placed on the palm and wrist of the left hand. The response was recorded on a Grass Model 5B polygraph.

Eight nouns, four concrete and four abstract, were presented to the $S$. The nouns were obtained from a dictionary of nouns rated on concreteness, imagery, and meaningfulness (Paivio, Yuille, \& Madigan, 1968). The four concrete nouns, bird, apple, star, and mountain, had high imagery ratings, while the abstract nouns, chance, theory, event, method, had low imagery ratings. The words were equated for frequency. Two different random-order lists were used.

\section{PROCEDURE}

After the GSR electrodes were attached to the $S, 5 \mathrm{~min}$ were allowed to elapse for the $S$ to get accustomed to the situation. After the $S$ heard the instructions, he was told to put his face against the support and fixate on a $1 \times 1$ in. plus $(+)$ sign located in the middle of the screen to keep eye movements at a minimum.

The tape-recorded stimuli were presented to the $S$ over earphones. The $S$ was required to form an image or mental picture to each word and to press the key when he had the image. The key press stopped a clock from which $E$ recorded the latency of image formation. Ten seconds were allowed for this task, after which the $S$ heard the word "relax." This was his cue to press the key if he had not already done so. Then he was to relax "mentally" until he heard the next word, which was presented after another 10-sec interval. The sequence, therefore, was: "relax" - "word" - "relax" - "word" - and so on.

\section{RESULTS}

Response Magnitude

Change scores were calculated for both the PR and GSR data. In the case of the pupillary response, the change score was the

Fig. 1. Pupil size during attempts to generate images to concrete and abstract nouns and during a preceding control period. Arrows indicate points of maximum dilation. 
difference between the largest diameter during the "imaging", period and the diameter in the last frame $(.5 \mathrm{sec})$ before stimulus presentation. The GSR change score was calculated by subtracting the maximum deflection (decrease in resistance) during the "imaging" period from the resting level during the last $1 / 2 \mathrm{sec}$ before presentation of the stimulus. ${ }^{2}$

Concrete and abstract nouns differed significantly in terms of mean pupillary change scores, with greater dilation $(\mathrm{t}=2.23, \mathrm{p}<.05)$ occurring to the abstract words (Fig. 1). The mean GSR change scores for concrete and abstract words did not differ significantly $(\mathrm{t}=1.12, \mathrm{p}<.10)$.

$$
\text { Response Latency }
$$

Key press, PR, and GSR latency scores were analyzed. Pupillary latency was the time to maximum dilation after stimulus presentation, and GSR latency was the time to maximum deflection. The mean key-press latencies for the two types of nouns differed significantly $(t=6.18, p<.001)$ with longer latencies occurring to the abstract nouns. Concrete and abstract nouns differed significantly in terms of mean pupillary latency, with longer latencies $(t=3.55, p<.01)$ occurring to the abstract nouns (Fig. 1). Significantly longer GSR latencies $(\mathrm{t}=2.49$, $\mathrm{p}<.05)$ also occurred to the abstract nouns. Correlations

The pupil and GSR change scores were converted to standard scores. A correlational analysis of mean scores over all stimuli for each $S$ revealed a significant $(\mathrm{df}=15, \mathrm{p}<.05)$ correlation of .50 between PR and GSR change scores and also a significant $(\mathrm{df}=15, \mathrm{p}<.05)$ correlation of .53 between PR magnitude and PR latency. Longer latencies were associated with larger pupil size.

\section{DISCUSSION}

The results support the conclusion that pupillary dilation is highly related to the "cognitive difficulty" of the imagery task. Both the magnitude and the latency of the pupillary response were significantly greater for abstract than for concrete words. These data are consistent with previous results (Paivio \& Simpson, 1968; Simpson, Molloy, Hale, \& Climan, 1968) in suggesting that pupillary latency may be more sensitive than magnitude as an indicator of cognitive task difficulty. The abstractness of the stimulus words accounts for $27 \%$ of the variance in PR latency scores while accounting for only $11 \%$ of the variance in PR magnitude scores.

The GSR does not seem to be as strongly related to degree of difficulty in image formation. The magnitude of the GSR did not significantly differentiate abstract from concrete nouns even though the GSR change score correlated with the PR change score. Kling \& Schlosberg (1961) reported results which suggest that GSR reflects attentional processes. These authors found increases in conductance when Ss were warned to get ready to work and again when warned to stop work and to rest. These responses would not habituate out and the authors concluded that each shift of attention from one phase to another of even a familiar task is marked by an increase in activation or arousal. This suggests that, in the imagery task, the GSR was reflecting shifts in attention from one word to the next. GSR latency, on the other hand, differed significantly for the two types of nouns, suggesting that, if GSR is reflecting an attentional process, it may somehow be related to the concrete-abstract dimension.

The results of the present investigation, then, suggest that pupillary dilation is more sensitive than the GSR to changes in cognitive activity occurring during an imagery task. In addition, they suggest that the latency of such physiological responses may be a more sensitive indicator than is the magnitude of these responses.

\section{REFERENCES}

BERRY, R. N. Skin conductance levels and verbal recall. Journal of Experimental Psychology, 1962a, 63, 275-277.

BERRY, R. N. Task difficulty, performance and skin conductance. Perceptual \& Motor Skills, $1962 \mathrm{~b}, 15,474-477$.

DAVIS, R. C. Modification of the galvanic reflex by daily repetition of a stimulus. Journal of Experimental Psychology, 1934, 17, 504-535.

HESS, E. H. Attitude and pupil size. Scientific American, 1965, 212, 46-54.

HESS, E.H., \& POLT, J. M. Pupil size in relation to mental activity during simple problem solving. Science, 1964, 143, 1190-1192.
KAHNEMAN, D., \& BEATTY, J. Pupil diameter and load on memory. Science, 1966, 154, 1583-1585.

KLING, J. W., \& SCHLOSBERG, H. Relation of skin conductance and rotary pursuit during extended practise. Perceptual \& Motor Skills, $1961,12,270-275$.

PAIVIO, A. Latency of verbal associations and imagery to noun stimuli as a function of abstractness and generality. Canadian Journal of Psychology, 1966, 20, 378-387.

PAIVIO, A., \& SIMPSON, H. M. The effect of word abstractness and pleasan tness on pupil size during an imagery task. Psychonomic Science, $1966,5,55-56$.

PAIVIO, A., \& SIMPSON, H. M. Magnitude and latency of the pupillary response during an imagery task as a function of stimulus abstractness and imagery ability. Psychonomic Science, 1968, 12, 45-46.

PAIVIO, A., YUILLE, J. C., \& MADIGAN, S. A. Concreteness, imagery, and meaningfulness values of 925 nouns. Journal of Experimental Psychology Monograph Supplement, 1968,76, No. 1.

SILVERMAN, S. J., COHEN, S. I., \& SCHMAVONIAN, B. M. Investigation of psychophysiologic relationships with skin resistance measures. Journal of Psychosomatic Research, 1959, 4, 65-87.

SIMPSON, H. M., MOLLOY, F. M., HALE, S. H. \& CLIMAN, M. H. Latency and magnitude of the pupillary response during an imagery task. Psychonomic Science, 1968, 13, 293-294.

YUILLE, J. C., \& PAIVIO, A. Latency of imaginal and verbal mediators as a function of stimulus and response concreteness-imagery. Journal of Experimental Psychology, 1967, 75, 540-544. NOTES

1. This research was supported by Grants APA-304 (to the first au thor) and APA-87 (to the second author) from the National Research Council of Canada, and a grant from the University of Western Ontario Research Fund.

2. No significant correlations were found between prestimulus level and maximum change scores for either PR or GSR.

\section{Counterconditioning, neutral conditioning and extinction effects for the meaning of nonsense syllables}

\section{ADAM MILLER, Saint Cloud State College, St. Cloud, Minn. 56301, and NORMA CLARK, University of Denver, Denver, Colo. 80210}

This study examined the effects of counterconditioning, neutral conditioning, and extinction, and different amounts of each, upon reshifting the rated meaning of nonsense syllables. For 120 Ss only counterconditioning was significantly effective at changing meaning.
Studies of the processes and conditions basic to change of word meaning have begun to identify a pattern among relationships. Using paired-associate learning procedures, the rated meaning of words, phrases, and nonsense syllables has been predictably changed. Designation of the change process as higher-order classical conditioning (Staats, 1968) has been accompanied by a series of studies delimiting the characteristics of the process and the variables related to it. One unexpected 